\title{
RED EMPRENDESUR: UNIVERSIDAD, EDUCACIÓN, EMPRENDIMIENTO E INNOVACIÓN
}

\author{
Pedro Vera-Castillo ${ }^{1}$ \\ ${ }^{1}$ Presidente Red Emprendesur, miembro oficial Foro Mundial del Emprendimiento \\ Profesor asociado, Facultad de Ingeniería \\ Director del Programa de Fomento y Desarrollo del Emprendimiento (Emprendo) \\ Universidad de Concepción, Concepción, Chile \\ Correo electrónico: pvera@udec.cl
}

Recibido: 15 de enero del 2015. Aprobado: 30 de marzo del 2015.

Cómo citar este artículo: P. Vera-Castillo. "Red Emprendesur: universidad, educación, emprendimiento e innovación". Ingeniería Solidaria, vol. 11, n. ${ }^{0}$ 18, pp. 57-63, ene.-dic. 2015. doi: http://dx.doi.org/10.16925/in.v11i18.991

Resumen. Este artículo de reflexión analiza cómo la Red Emprendedorismo e Innovación en América Latina (Emprendesur) potencia el trabajo en conjunto de todos los actores comprometidos con el desarrollo de ecosistemas regionales o locales de emprendedorismo e innovación con énfasis en la educación y la formación de emprendedores. A partir de la conocida formulación del ecosistema emprendedor propuesta por David Isenberg de Babson College, se defiende la idea acerca de cómo los dominios de capital humano y cultura son los que están en el centro del ecosistema emprendedor. Se postula entonces que son precisamente los dominios de capital humano y cultura los que están en el centro del ecosistema emprendedor. Su naturaleza y la calidad de las interacciones entre ellos, y en relación con los demás dominios que los rodean, serán responsables de generar o no el emprendimiento durable que se busca. Como conclusión, se propone actualizar la propuesta de Isenberg para aplicarla al contexto latinoamericano y avanzar en la conceptualización de ecosistemas emprendedores durables que vinculen activamente educación, emprendimiento e innovación con un enfoque nuevo, el cual se debe construir entre todos.

Palabras clave: ecosistema, educación, emprendimiento, universidad. 


\title{
EMPRENDESUR NETWORK: UNIVERSITY, EDUCATION, ENTERPRISE AND INNOVATION
}

\begin{abstract}
This article of reflection analyzes how the Red Emprendedorismo e Innovación en América Latina (Emprendesur) boosts joint work by all actors committed to developing regional or ecosystems for enterprise and innovation with an emphasis on education and training for enterprising individuals. Based on the well-known formulation of the entrepreneurial ecosystem proposed by David Isenberg of Babson College, the article defends the idea of how human capital and cultural domains are at the core of the entrepreneurial ecosystem. It argues that the human capital and cultural domains are at the center of the entrepreneurial ecosystem. Their nature and the quality of the interactions between them, and in relation to the other surrounding domains, are responsible for generating or failing to generate the desired durable enterprise. The article concludes by proposing to update the Isenberg formulation for application in the Latin American context and advance in the conceptualization of durable entrepreneurial ecosystems that actively link education, enterprise and innovation using a new approach that must be created by all.
\end{abstract}

Keywords: ecosystem, education, enterprise, university.

\section{REDE EMPRENDESUR: UNIVERSIDADE, EDUCAÇÃO, EMPREENDIMENTO E INOVAÇÃO}

Resumo. Este artigo de reflexão analisa como a Rede Empreendedorismo e Inovação na América Latina (Emprendesur) potencializa o trabalho em conjunto de todos os atores comprometidos com o desenvolvimento de ecossistemas regionais ou locais de empreendedorismo e inovação com ênfase na educação e na formação de empreendedores. A partir da conhecida formulação do ecossistema empreendedor proposta por David Isenberg de Babson College, defende-se a ideia sobre como os domínios de capital humano e cultura são os que estão no centro do ecossistema empreendedor. Postula-se, então, que são precisamente os domínios de capital humano e de cultura os quais estão no centro do ecossistema empreendedor. Sua natureza e a qualidade das interações entre eles, e com relação aos demais domínios que os rodeiam, serão responsáveis de gerar ou não o empreendimento durável que se procura. Como conclusão, propõe-se atualizar a proposta de Isenberg para aplicá-la ao contexto latino-americano e avançar na conceitualização de ecossistemas empreendedores duráveis que vinculem ativamente educação, empreendimento e inovação com uma abordagem nova, a qual se deve construir entre todos.

Palavras-chave: ecossistema, educação, empreendimento, universidade. 


\section{Introducción}

La Red Emprendedorismo e Innovación en América Latina (Emprendesur) [1] reúne hoy a más de 300 académicos, profesionales e investigadores de Argentina, Bolivia, Brasil, Colombia, Chile, Ecuador, México, Paraguay, Panamá, Perú, Uruguay, República Dominicana y Venezuela. Representamos una red que, de manera voluntaria, invita a la colaboración y al trabajo conjunto a académicos, investigadores, profesionales del sector público, empresarios, educadores y a todos aquellos interesados en la formación de emprendedores, así como en el fomento y apoyo al desarrollo del emprendedorismo y la innovación en todos sus niveles y en todas sus dimensiones.

La red no pretende ser ni construir un modelo. Se invita sólo a compartir las buenas prácticas y a respetar cada uno su cultura y su contexto local. Así, no se quiere sólo ser parte, sino que se aspira a animar decididamente la consolidación de ecosistemas de emprendimiento durables en nuestra región, promoviendo el encuentro, la reflexión y el debate participativo y colaborativo.

\section{El desafío es construir un ecosistema emprendedor durable adaptado a nuestra cultura $y$ a nuestros entornos}

Diversos estudios internacionales [2] y la experiencia de la Red de Emprendedorismo e Innovación en América Latina (Emprendesur) lo confirman: es necesario dar un salto muy importante en emprendimiento y comprometer a porcentajes claramente más significativos de la población económicamente activa si queremos generar mayor riqueza, distribuirla de manera más equitativa, crear más y mejores empleos (especialmente para los jóvenes), y contar con futuras generaciones apasionadas de vivir y hacer crecer nuestros países, y no frustradas o decepcionadas con su desarrollo profesional y su vida personal. Esto explica en parte, por ejemplo, el importante apoyo que desde las políticas públicas se empieza a dar a las startups de rápido crecimiento [3].

Desde que se atendió la convocatoria de la Universidad Nacional de Luján de Argentina, en el 2004, con el fin de reunirse para constituir esta Red Emprendesur, se ha acumulado un saber hacer significativo, en relación con una nueva pedagogía emprendedora que debe cambiar la tradicional enseñanza universitaria en el contexto de una visión amplia y sistémica sobre las dimensiones y los elementos del proceso emprendedor. Es gratificante a este respecto ver que la educación y la formación de emprendedores empiezan a ser objeto de creciente interés, así como de estudios específicos [4].

Para que dicho cambio cultural sea sustentable, se comparte con Fernando Dolabela la idea según la cual el proceso liberador del desarrollo de las competencias emprendedoras debe comenzar "desde la más tierna infancia", es decir, desde los niveles iniciales del sistema escolar formal [5].

Se ha entendido perfectamente, por lo demás, cómo el emprendimiento no es un tema sólo de la academia, sino que tenemos el deber de trabajar en red con todos los actores regionales vinculados al desarrollo de la innovación y el emprendimiento. Esto es, con todos los actores de la comunidad nacional y de la comunidad internacional comprometidos con el desarrollo emprendedor.

De acuerdo con la propuesta de David Isenberg de Babson College [6], un Ecosistema Emprendedor podría representarse como la interacción de seis dominios, según se ve en la figura 1 .

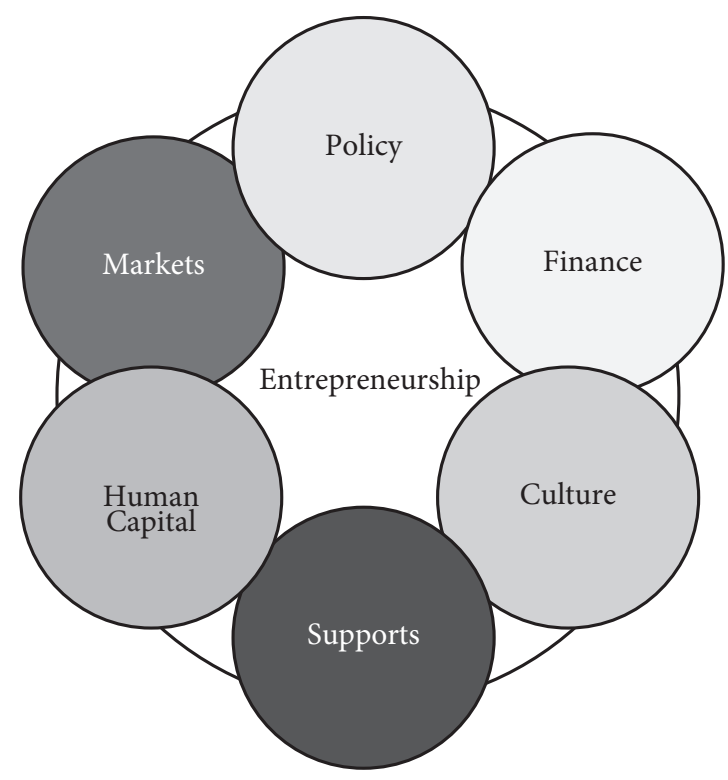

Figura 1. Ecosistema de emprendimiento según Isenberg Fuente: [6] 
En el dominio de las políticas se hace referencia principalmente a instituciones de gobierno central, regional y local, capaces de propiciar un entorno regulador que incentive la actividad emprendedora.

En el dominio financiero será particularmente relevante la actuación de instituciones de microcréditos, inversionistas ángeles y capital de riesgo, los cuales faciliten el acceso al capital semilla a diferentes segmentos de emprendedores.

En el dominio cultural se requieren normas sociales comúnmente aceptadas de tolerancia al riesgo y al fracaso, las cuales incentiven la innovación y la creatividad, reforzadas por las historias de éxito - particularmente las locales-que incentiven modelos positivos de desempeño.

El campo de los servicios incluye la infraestructura de telecomunicaciones, transporte y energía, así como la existencia de parques tecnológicos, distritos industriales o zonas francas, y la disponibilidad de servicios profesionales de apoyo a las nuevas iniciativas en los campos legal, laboral, fiscal o tecnológico.

El dominio del capital humano se refiere a la capacitación de la fuerza laboral, el apoyo del sistema educativo en sus diferentes niveles y la difusión de la cultura emprendedora y de competencias específicas para emprender.

En cuanto a los mercados, la existencia de clientes exigentes y capaces de asumir riesgos en la adopción de nuevos productos y servicios, resulta tan relevante como un juego competitivo transparente. El emprendimiento, de acuerdo con Isenberg, sería entonces el resultado de la acción coherente y las redes de sinergias creadas entre estos dominios.

\section{Hacia una propuesta renovada}

Si bien en el trabajo citado y en todo el importante aporte investigativo al tema del emprendimiento que ha desarrollado, Isenberg señala con razón que cada ecosistema de emprendimiento es único, no es menos cierto que a menudo se cita y se refiere con carácter paradigmático tal modelo como el que debemos emular de manera mecánica. En ese marco, algunas ideas fuerza de Emprendesur pueden contribuir a una reflexión complementaria y renovada de esta propuesta. Las sintetizamos así:
- Se necesita una nueva educación que libere y potencie las competencias de las personas, de manera que estén en capacidad de concretar sus sueños a través del emprendimiento y la innovación. Se trata no sólo de una nueva educación superior, sino más que eso y antes que eso, una nueva escuela, profesores preparados para poner en marcha innovaciones pedagógicas desde la educación básica. En otras palabras, el acento no puede seguir puesto sólo en políticas públicas que apunten al fomento y apoyo a emprendedores y a la innovación, si no enfrentamos primero los cambios radicales que requieren nuestros sistemas educativos.

- Requerimos una mirada integral y humanista sobre el emprendimiento. Este puede desarrollarse en todos los ámbitos y dimensiones de la vida profesional y personal: en lo productivo, en lo social, en lo cultural, en lo educacional.

- Deseamos nuevos emprendedores que generen nuevos emprendimientos, pero también las organizaciones, las instituciones y las empresas requieren hoy el emprendimiento y la innovación en su interior con el fin de renovarse y subsistir en este escenario global dominado por la competencia: es la tarea de los intraemprendedores o emprendedores corporativos. Todo nuevo técnico y profesional requerirá entonces hoy de nuevas competencias y habilidades que complementen y hagan pertinente el conocimiento.

- Sin embargo, nuestro enfoque es más amplio que el de sólo una nueva formación: desarrollar hoy las competencias para el emprendimiento y la innovación es, en nuestra opinión, la manera de empoderar a las personas en una sociedad del siglo Xxi turbulenta, impredecible y compleja. Ningún cuestionamiento ideológico podría alzarse frente a esta voluntad de hacer a las personas dueñas de su propio destino; empoderar a las personas es justamente el fundamento y el objetivo para una mejor y más amplia democracia.

- Y, por último (pero no menos importante), consideramos que el emprendedorismo y la innovación deben romper las exclusiones sociales. No sólo abriendo las oportunidades a emprendedores de todos los estratos sociales, sino contribuyendo a la distribución de la riqueza, cuya inequidad sigue siendo una vergüenza y un talón de Aquiles que frena el desarrollo de nuestros pueblos. 
Así pues, hoy en día, la mirada está puesta en la construcción de los ecosistemas emprendedores en los cuales la ciudad, sus autoridades y sus habitantes juegan un rol central. Pero por otra parte es claro que este esfuerzo no debe agotarse en, ni limitarse a, los emprendimientos dinámicos de alta tecnología.

La hipótesis propuesta es quela decisión dehacer que el emprendedorismo y la innovación estén en el centrodelaculturadenuestrassociedadesnopasasolamente por el desarrollo de políticas públicas enfocadas en favorecer el desarrollo de empresas dinámicas de alta tecnología, sino que se requieren nuevas políticas educativas complementadas por la creación de sistemas amplios y suficientemente diversificados de apoyo e incubación, los cuales permitan el desarrollo del potencial emprendedor de todos los actores de la sociedad. Desarrollar este potencial no es hoy sólo requerimiento del desarrollo económico, sino que es, como se ha mencionado, una condición de ciudadanía en la sociedad del siglo xxI.

Con el fin de mantener en este artículo la nomenclatura propuesta por Isenberg, y con el propósito de facilitar la comprensión de los enfoques que aquí se tratan, se postula entonces que son precisamente los dominios de capital humano y cultura los que están en el centro del ecosistema emprendedor. Su naturaleza y la calidad de las interacciones entre ellos, y en relación con los demás dominios que los rodean, serán responsables de generar o no el emprendimiento durable que se busca (figura 2).

El hecho de que en diferentes países y regiones se hayan generado sólidos ecosistemas de emprendimiento en ausencia, o "sin esperar" el desarrollo de una educación pertinente para el emprendimiento y la innovación - por ejemplo, Israel o Silicon Valley

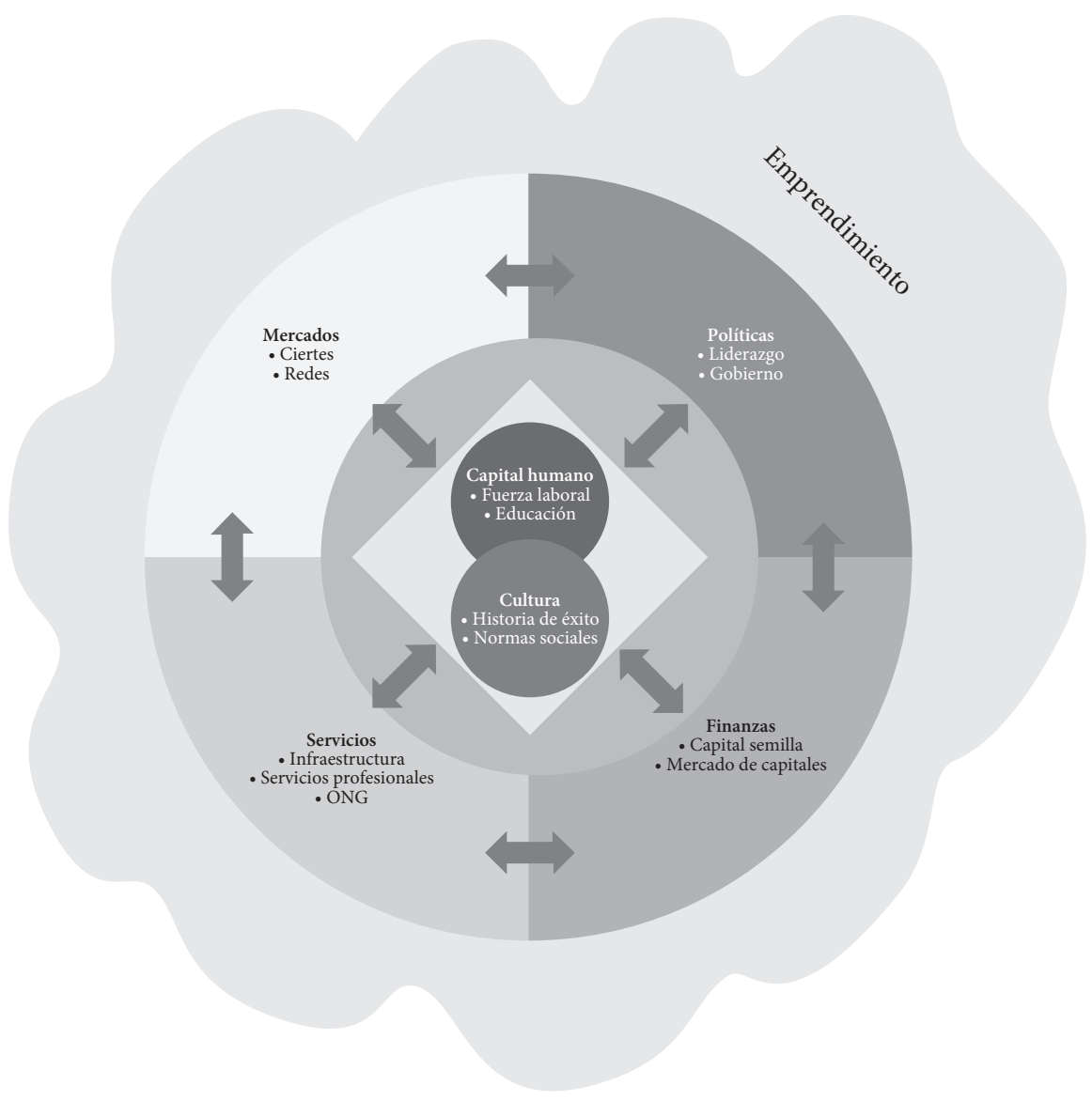

Figura 2. Ecosistema emprendedor durable 
[7]—, sugiere que es necesario considerar de manera flexible estas modelizaciones y ligarlas siempre a las características fuerza de la cultura y el territorio locales.

Ello se explica, además, porque para el desarrollo de un ecosistema emprendedor son ciertamente relevantes la calidad de las relaciones, los desarrollos paralelos y las sinergias entre los dominios mencionados, sin que a priori pueda asignarse a un dominio o a uno de sus elementos un carácter decisivo y dominante.

Sin embargo, se postula como en el caso de América Latina, justamente "el carácter único de nuestros ecosistemas de emprendimiento", se caracteriza porque estamos a tiempo y requerimos como condición esencial de la durabilidad a la que aspiramos que las políticas públicas pongan el acento, justamente, en la educación y la formación de emprendedores. Dicho de otro modo, en el capital humano y la cultura.

Siguiendo esta misma lógica, y poniendo el énfasis en la cultura, por ejemplo, la Comisión de las Comunidades Europeas [8] compara la cultura europea y la norteamericana, y concede un rol relevante al desarrollo de la "educación para el espíritu empresarial".

Esta formulación se entiende mejor en la óptica de visiones holísticas y sistémicas acerca del emprendimiento, concebido como condición de ciudadanía que permea todas las organizaciones y todos los sectores, y no sólo como la capacidad de generar negocios, que es justamente la visión de Emprendesur.

La universidad, por su rol en la sociedad, su compromiso con el desarrollo del conocimiento y la formación de ciudadanos emprendedores e innovadores, así como por su capacidad de convocatoria, está llamada a jugar un papel decisivo en la consolidación de estos ecosistemas de emprendimiento durables.

Para enfrentar la tarea de liderazgo planteada, sin embargo, se propone que la universidad latinoamericana se mire a sí misma y se renueve radicalmente, teniendo en cuenta lo siguiente:

- Desde luego, constituirse a sí misma como un ecosistema participativo en que sus actores se sientan comprometidos con la definición y la ejecución de su misión institucional.

- Introducir el desarrollo y el fortalecimiento de las competencias para emprender e innovar en todas las mallas curriculares de la formación profesional, de manera transversal e interdisciplinaria.

- Crear mecanismos concretos de incubación y apoyo para estudiantes e investigadores que deseen emprender en cualquier área.

- Potenciar sus mecanismos de transferencia de conocimiento al medio y de protección de la propiedad intelectual.

- Ligar su investigación con el desarrollo regional y local.

- Potenciar su inserción en el medio local, nacional e internacional, con una nueva concepción de lo que hoy día denominamos la Tercera Misión.

- Evaluar su trabajo y su aporte a la sociedad con nuevos indicadores de impacto, y no sólo con los criterios ligados tradicionalmente a la investigación científica, como son, por ejemplo, los papers (artículos en revistas de corriente principal), cuyo impacto real está hoy día siendo cuestionado por diversos estudios internacionales [9].

\section{Conclusión}

Por ahora, a partir de la propuesta de Isenberg acerca de los pilares básicos del ecosistema emprendedor, se propone actualizar su formulación aplicándola a nuestro contexto latinoamericano, y avanzar en la conceptualización de ecosistemas emprendedores durables que vinculen activamente educación, emprendimiento e innovación con un enfoque nuevo, el cual se debe construir entre todos.

\section{Referencias}

[1] Red Emprendesur, Declaración de Principios Red Emprendesur. [En línea]. Disponible en: http://emprendesur.net. Último acceso: marzo 2, 2015.

[2] GEM 2014 Global Report. [En línea]. Disponible en: http://www.gemconsortium.org/ docs/3616/gem2014-global-report. Último acceso: marzo 3, 2015.

[3] oCDE, Startup América Latina: Promoviendo la innovación en la Región, Estudios del Centro de Desarrollo, París, Francia: OECD Publishing, 2013. [Enlínea].Disponible en: http://dx.doi.org/10.1787/9789264202320-es. Último acceso: marzo 19, 2015. 
[4] Global Entrepreneurship Monitor Special Report, Una perspectiva global sobre la educación y la formación emprendedora, Babson College, Univ. del Desarrollo, Univ. de Reykjavik. [En línea]. Disponible en: http://www.gemconsortium.org/docs/ download/275. Último acceso: noviembre 12, 2014.

[5] F. Dolabela, Pedagogía emprendedora: la enseñanza de emprendedorismo en la educación básica, en busca del desarrollo social sustentable. Una experiencia latinoamericana, $1^{\text {a }}$ edición, Argentina: Ed. Santa Fe Innova, 2010.

[6] D. Isenberg, The Entrepreneurship Ecosystem Strategy as a New Paradigm for Economic Policy: Principles for Cultivating Entrepreneurship, 2011. [En línea]. Disponible en: http://www.wheda.com/uploadedFiles/Website/About_Wheda/ Babson\%20Entre-
preneurship\%20Ecosystem\%20Project.pdf. Último acceso: noviembre 26, 2014.

[7] D. Isenberg, What an Entrepreneurship Ecosystem Actually Is, 2014. [En línea]. Disponible en: https:// hbr.org/2014/05/what-an-entrepreneurial-ecosystem-actually-is/. Último acceso: marzo 10, 2015.

[8] Comisión de las Comunidades Europeas, Libro Verde: El Espíritu Empresarial en Europa. Bruselas: DG Empresa. [En línea]. Disponible en: http://www.oei. es/etp/ green_paper_final_es.pdf. Último acceso: marzo 9, 2015.

[9] E. Bueno y F. Casani, "La tercera misión de la Universidad: enfoques e indicadores básicos para su evaluación", Revista Economía Industrial, n. ${ }^{\circ}$ 366, pp. 43-59, 2007. 